\section{VOYAGE DU MINISTRE DES TRAVAUX PUBLICS AUX INSTALLATIONS HYDRAULIQUES DES ALPES}

M. Millerand, Ministre des Travaux publics, ayant accepté l'invitation que lui avait faite la Chambre syndicale des Forces hydrauliques, de visiter les installations créées dans les Alpes pour la production de l'énergie électrique, son transport, sa distribution et son utilisation par les industries de l'ćlectrométallurgie et de l'électrochimie, s'est rendu à Avignon, le s4 août rgro. Il était accompagné de M. Henriot, Inspecteur général des Mines, Directeur des Mines et des Distributions d'énergie électrique au Ministère des Travaux publics, et de M. Marlio, Maître des Requêtes au Conseil l'Etal, Chef du cabinel technique au même Ministère. M. Millerand fut reçu, à son arrivée, par MM. Cordier, Présidenl ; Michoud, vice-président, et R. Pinot, secrétaire général de la Chambre syndicale.

Malgré le lemps relativement considérable que M. Millerand avait eu l'obligeance de réserver pour celle tournée, il ćlail malériellement impossible de lui faire voir, en quatre journées, toutes les installations et toutes les usines qui, par lingéniosité de leurs aménagements et la puissance de leur production auraient mérité d'altirer son attention. D'ailIcurs, la Chambre syndicale se trouvait forcée de limiter son programme à la visite de quelques installations et de quelques usines, susceptibles de donner une vue d'ensemble sur les industries qu'elles représentent ; M. le Ministre des Travaux publics devait, en effet, consacrer nécessairement une journée enlière à l'étude de différents projets intéressant le lomaine public, qui procureront, par leur réalisation, un nouvel cssor aux industries hydrauliques, à l'agriculture provençale, cl une nouvelle source de richesse au pays enlier.

la première journée fut occupée par l'examen de ces projuls, que présenta, à M. Millerand, M. de la Brosse, Ingénieur en chef des Ponts et Chaussées, chargé du service hydraulique de la région des Alpes. M. de la Brosse eut aussi l'occasion, au cours de ce voyage, d'altirer l'altention de M. Ie Ministre des Travaux publics sur les postes de jaugeage qu'il at installés dans toute la région, et de lui signaler les grands services rendus par ces derniers tant à l'Elal qu'à l'industrie privée, au point de vue d'une meilleure ulilisalion de leurs domaines respectifs.

A Serre-Ponson, M. le Ministre s'arrêta pour étudier le projel du barrage de la Durance qui, s’il était réalisé, assurerait l'irrigation complete el mélhodique de la Provence el domerail à l'industric une disponibilité de 45.000 IIP ( $)$. Les explications lui furent domnées par M. Wilhelm, Ingénieur en chef du département des llantes-Alpes, linfaligable aptitre de ce projet de barage, qui ne tend ì rien moins qu'à créer, dans celte région, un lac égal à celui du Bourget.

Au cours de celle loumée qui s'élendit sur qualre dépinInments, lous les travaux faits on à faire pour assurer le meilleur rendement possible dans l'emploi de la houille blanche furenl présentés au Ministre par les chefs de service des Ponts el Chaussées de ces divers départements, par M. I'Tnspecteur général Rivoire-Vicat ; par MM. Wilhelm, Domergue, Golleland, et par les Présidents de Gonseils ef les Direcleurs des différentes Sociélés hydro-électriques.

Il nous est impossible de reproduire ici l'exposé que les chefs des usines visitées firent au Ministre el à sa suile; des travaux qu'ils avaient fails ol des résultats auxpuels ils sont arrivés. Il nous suffira, pour bien montrer l'étendue du

(*) Voir La llouille Blanche de mars 1910 , page 73 . champ qu'il fut donné à $M$. le Ministre des Travaux publics de parcourir, d'indiquer le programme de la lournée qu'il a accomplie, el nous ne saurions traduire plus exactement l'impression que M. Millerand et ses collaborateurs ressentirent au cours de ces visites, qu'en reproduisant le discours qu'il prononça à Grenoble, lors du banquet offert par la Cinambre de Commerce.

Voici quel fut le programme du voyage accompli par M. Millerand, les $25,26,27$ et 28 aoùt I9ro :

I Jounnée. - Matinée. - Perthuis, Mirabeau.

Après-midi. - La Brillanne : Usine de l'Energie électrique du Littoral Médilerranéen. - Ventavon : Usine de la Société des Forces Motrices de la Haute-Durance. - SerrePonson : Projet de barrage.

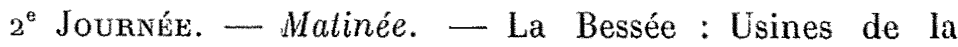
Société Electrométallurgique française.

Après-midi. - Livet : Usines de la Société Keller-Leleux. - Les Glavaux : Usines de la Compagnie Universelle d'acétylène et de la Société d'Electrochimie. - Gavet : Usine de la Société Grenobloise de Force et de Lumière.

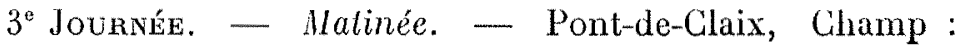
Usines de la Société électrique de Fure et Morge et de Vizille. -- Jouchy : Visine de l'usine. - Déjeuner de la Chambre de Commerce.

Après-midi. - Saint-Jean-de-Maurienne : Usine de la Compagnie des Produils Chimiques d'Alais et de la Curmargue.

$4^{\circ}$ Jounnée. - Matinée. - Saint-Michel : Usine de Calypso de la Compagnie des Produits Chimiques d'Alais el de la Camargue. - Ugine : Usines de la Sociélé Electromélallurgique Paul Girod.

Après-midi. - Le Fayel : Usine l'aul Girod.

Nous donnons, ci-dessous, le texte des discours prononcés à l'issue du banquet offert à $M$. le Ministre des Travaux publics par la Chambre de Commerce de Grenoble, le 26 aoùt r9ro, par MM. Brénier, Président de la Chambre de Commerce ; Cauwes, secrétaire général de la Préfeclure de l'Isère, représentant le Préfel de l'Isère, absent; Cordier, présidenl de la Chambre syndicale des Forces hydrauliques, cl enfin, par M. le Ministre des Travaux publics.

\section{Discours de M. Brenier}

"Monstedir tak Ministre,

"Jo ne veux pas abuser de los inslanls, que rolre activilé bien conmue rend doublement précieux ; mais je tiens à vous remercite d'avoir bien voulu accepter la sinple invilation de notre Chambre de Commerce a ex léjounte inlime.

"Nous avons fons le sonvenic de lit sollicifude que, comme Ministre du commeree, vors témoignie\% à la frospérité du Commere et de l'Industric.

" Cetle sollicitude, vous voule\% bien nous la manifesler encore, comme Ministre des Travaux publics, par l'intêt que vous prenez aux grands travaux effectués dans notre région pour l'aménagement des forces hydrauliques. Celle permanence d'un inlérêt qui nous tonche profondémont n'a rien qui puisse surprendre. En effet, loul se lient dans la nalure : Commeres, Industrie, Agricullure, Sciences, Thavaux publics et privés, qui tous concourent au bien-être social.

"Notro Université elle-mème, n'est pas restéc étmangère au grand mouvement industriel qui s'tas produit dans le Dauphiné. Elle l'at provoqué du resle en grande parlic par la création el le développement de son Institut b́lectrotechniqu, qui étend ses enseignements at développe son aclivile dans le domaine los principales industrits de la région. Let choix de Crenoble pour siège de es véribble Instibut Polylechnique no pouvait done etre plus heureux ì tous les points de we.

"De plus qualifiés que moi vous ont déjà donmé, vol vous donneront

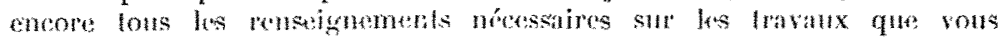
visite\% ; ces travaux vons laissoront certatinement une impression des plus favorables et la plus propere ì faire nâtle les encouragements allendus te volre Ministire pour l'utilisation des conrs d'enu du domaine de l'Tfat. 
“ Je pourrais, Monsieur le Ministre, vous entretenir de diverses autres questions qui intéressent la Cihambre de Commerce, mais ce serait sortir du but de votre voyage, el du caractère que vous avez voulu donner $\grave{a}$ ce déjeuner. Permettez-moi seulement d'ajouter que le mouvement d'affaires déjà produit dans notre région par l'utilisation des forces hydrauliques justifie les prévisions de notre Chambre de commerce, qui prend de jour en jour plus d'importance. Pour favoriser ce mouvement, clle a fait construire un édifice qui donne déjà satisfaction à tous ses services, et qui va se compléter en ce moment par l'édification d'une Bourse de commerce et de valeurs.

"Tous les membres de notre Chambre apportent à leur Président un concour's actif et vigilant ; ils sont heureux par Jeur présence de vous témoigner l'unanimité de vues qui constitue la règle de notre Compagnie pour le bien public.

“ Messieurs, en vous priant de vous joindre à moi pour adresser l'assurance de notre dévouement à M. Fallières, le respecté Président de la République, je lève mon verre à la santé de M. Millerand, Ministre des Travaux publics, à celle de M. le Secrétaire Général, représentant M. le Préfet de l'Isère, que j'ai le regret de savoir retenu loin de nous par une indisposition, enfin à celle de M. Cordier, l'éminent Président de la Chambre Syndicale des Forces hydrauliques, et de tous ses dévoués collaborateurs $)$.

\section{Discours de M. Cauwes}

\section{“ Monsieun le Ministre,}

"Dans ce pays, berceau de la houille blanche, où les forces de la nature ot l'énergie des caractères s'allient si merveilleusement pour produịre de la richesse, - dans cette région où des initiatives intelligentes ont fait naître tant d'industries nouvelles s'ajoutant à celles qui de tout temps ont fail la renomméc du Dauphiné, - dans ce département de l'Isère aux sites ravissants qui, avec le concours de l'Etat, des communes et des Sociétés locales, améliore sans cesse ses moyen de communication pour draîner toujours davantage le tourisme et pour développer le commerce, votre visite d'études constitue le plus précieux des encouragements.

"Je tiens donc a mon tour, au nom de l'Administration préfectorale, à vous remercier d'ivoir accordé à l'Isc̀re la favcur d'un séjour trop bref au gré de tous.

"Permettey-moi de remercier aussi M. le président Brenier, et la Chambre de commerce tont entière, d'avoir associé la Préfecture de l'Isèro à la manifestation de respectueuse sympathie dont vous êtes l'objet, et d'exprimer ici les excuses et les regrets de M. Ie préfet Boncourt, que son état de santé tient encore éloigné de Grenoble.

"M. le frésident Brenier vous a dit d'excellentes ehoses, Monsieur le Ministre, avec tout son cœur ; je suis certain d'être l'interprète fidèle de la pensée de tout le monde ici en ajoutant à son discours quelque chose qu'il me reprocheri dans sa modestie : mais il est juste que vous sachiez que M. Brenier n'est pas sculement le président de la Chambre de commerce, il en est l'âme ; il en est le génic bienfaisant ; c'est lui qui a contribué de ses deniers ì élever le palais de cette Compagnie et qui, à l'heure actuelle, agit avec le même, résintéressement pour réaliser la création de la Bourse de Commerce de Grenoble ; c'est lui aussi qui, par un don généreux, a fait naître récemment l'Ecole de papeterie de cetle ville; c'est lui enfin que nous retrouvons dans toutes les ouvres de solidarité comme le plus averti des philanthropes.

"Laissez-nous espérer, monsicur le Ministre, que vous garderez de volre passage dans l'Isère des impressions qui vous inciternnt à revenir parmi nous, tout à fait officiellement cette fois. comme hôte du peuple dauphinois tout entier. En attendant, yous conserverez sans donte un souvenir réconfortant de la vigueur et de la générosité do la démocratie de ce département, quand vous saurez que votre hôte, M. Brenier, qui en sort, s'est ćlevé avec ses seules forces à sa haule situation industriclle ef sociale, et qu'à l'heure actuelle, il consacre son temns et sa fortunc à secourir les travailleurs qui débutent et les déshérités de la vie.

"Je leve mon verre à M. Brenier et à la Chambre de commerce de Grenoble.

"Et au nom de l'Administration préfectorale, an nom de vos chefs de services départementaux et régionaux : MM. I'inspecteur général des ponts et chaussés. l'ingénieur en chef du service des fores hydranliames, l'ingénieur dos mines, le dirceteur des nostes et des télégraphes. je porte votre santé, monsieur le Ministre des Travaux publics. en vons donnant l'assurance que vos excellents collaboratewrs of la Préfecture de l'Iş̀re sont en tontes circonstances inspirés par Ic désir de bien servir la Républiqué el de la faire aimer ".

\section{Discours de M. Cordier}

\section{"Monsieun le Ministre,}

"Le Syndicat des Forces hydrauliques est né ì Grenoble. en rnor. Il a fait ses premières armes au Congrès de la Houille blanche. en rnos,

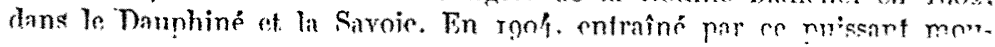

vement de centralisation industrielle que nous déplorons tous, mais qui est la conséquence nécessaire de la centralisation administrative et de la centralisation financière, il fixa ses pénates à Paris.

"Mais il laissa à Grenoble une partie de lui-même dans le Secrétariat qu'abrite le Palais de la Chambre de Commerce. Il y laissa ses plus ancicns et fidèles membres. Il y laissa le culte de son Président-Fondateur, mon regretté ami, M. Charles Pinat, qui lui traça la voie large et libérale où nous nous efforgons de suivre sa tradition. Il y laissa aussi cetle affection profonde que les Dauphinois et les Savoyards conservent toujours pour leur pays d'origine, affection qu'appellent irrésistiblement non seulement la beauté des montagnes, leur ciel pur, leurs fleurs embaumées, leurs neiges éternelles, mais aussi la vaillance, la discipline, l'esprit d'initiative les hommes qui en habitent les vallées.

"Aussi, Monsieur le Président, et Messieurs les Membres de la Chambre de Commerce, notre Syndicat tient-il à vous remercier bien sincèrement de nous avoir permis, par ce déjeuner, si aimablement organisé, de saluer M. le Ministre des Travaux publics, à Grenoble, capitale de la bouille blinche, et de lui dire combien nous lui sommes reconnaissants d'avoir entrepris un long et fatigant voyage pour se rendre compte, sur place, des besoiñs, des dosiderata, des résultats de nos industries :

" Ilonneur qu'on mérite valat double ", dit un vieux proverbe.

"Nous avons la conscience, Monsieur le Ministre, que nos industrics méritent 1 'honncur que vous voulez bien leur faire.

"Elles le meritent par le courage, par la persévérance, par la confiance (faut-i! dire l'optimismé ?) qui sont nécessaires pour l'aménagement des grandes forces hydrauliques.

“ Elles le méritent par la force qu'elles apportent au pays, en atténuant dans une large mesure son insuffisance de production houillère.

" Elles le méritent par les serviecs nombreux qu'elles rendent à l'agriculture ct à l'industric, en leur fournissant la force à bon marché, en 'aur apportant des produits que, seule, la houille blanche, permet de produire ćconomiquement.

"Flles le méritent par les améliorations de toute nalure qu'elles apportent dans la vie indiviluelle el dans la vie collective.

"Nous srmmes heureux, Monsicur Je Ministre, que vous ayez pu vous rendre compte, sur place, de nos efforts, et nous avons confiance que, de votre voyage d'études, vous emporterez de li sympathie pour les travailleurs de la houille blanche. Soyez assuré qu'ils conserveront avec gratitude le souvenir de votre visite, et permettez-moi d'être leur interprète à tous en portant de tout cœur votre santé, à laquelle je vous demande d'associer celle de M. le Président de la Chambre de Commerce Brenicr ".

\section{Discours de M. Millerand}

\section{"Messieuns,}

"Laissez-moi tont d'abord remercier les oratcurs précédents de leur Irop aimable accueil : $M$. Ie Scerétaire Général Cauwès. qui porte dignement un nom ionoró dans la science ćconomique et dans l'Injversité ; M. Ie Président Brenier. dont il vient d'être fait un óloge justifić qui reste encore an-dessons de ses mériles : M. Cordier, enfin, qui a si parfaitement organisó une tournée aussi instructive qu'agréable.

"Au cours de ces frois journées, j’ai éprouvé un enchantement constant en présence des spectacles naturels et des sites variés, tanfôt grandioses et tantôt charmants, que l'on ne connaît pas encore assez, el qui ne doivent pas ctre senlement la parure de notre pays, mais aussi l'une des sources de sa richesse.

"Des symdicals d'initiative comme celui de Grenoble et du Dauphiné. des associations sportives comme is Touring-Club, ont fait beawcoup pour amener dans ens régions pittoresques nos compatrioles el les étrangers ; il resle encore beaucoun à faire. C'est pour cela que. m'inspirant d'exemples nuicés au dehors, j'il voulu. dìs mon arrivée an Ministère des Travaux publies, non pas contrarier les initiatives privécs, ni m'y substituer. mais les grouner, coordonner et ainsi contupler lenrs efforts. et j'a obtemu du Parlement l'autorisation de eréer un Office National du Tourisme, qui sera constitas dans auelques jours, et formera un organe d'union entre syndicats ol assoniations.

"Mais le fourisme n'étnit. si is nuis dire, que la récomnense du vovage. Son but était d'initier lo Ministre à la mise en valeur des foress naturelles. de lui faire connâ̂lre le maşr et l'avenir de l'industrie hydraulirme. Je reviens émerveillé. parce qu'il n'est rien de tel pour connâtre los hommes at. les choses que d'entrer en contact direct avec enx. In vovage de milloues jours - aves de bons guides - fait plus nowr renseianer un Ministère que l'étude de longs rapports et de volumineux dossicrs.

"Je remercie les organisateurs de celle tournés de m'avoir permis d'anprécier te dévelonnement de cette inductrie oni nrésage yne révolutinn éronomiare. nuisqu'clle ne se horne plus a l'wlilisation losale de l'énergie. mais morlle róalise le transnort anx grandrs disfanees de la fore et de la lumiere. Il nst extranordinaire do songer que: dans un

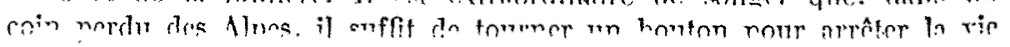


l'une grande ville à des centaines de kilomètres. C'est un exemple étonnant de la puissance de l'homme sur la nature, et qui présente un contraste surprenant entre ces installations et les effets qu'elles peuvent preduire. Dans une grande salle tournent quelques machines ; peu ou pas l'ouvi'iers. Entre de grands murs, analogues aux cages des fauves, dorment des forces plus dangereuses que les lions de la ménagerie. Le sentim+nt de les avoir assouplies et domesliquées éveille dans l'esprit humain un orgueil légitime, en même temps que la pensée de leur révolte, possible et lerrible, en nous faisant entrevoir des catastrophes, nous ramine à la modestie, et nous pousse à la recherche de nouvcaux progrès in point de vue économique et social.

"Pour lit réalisation de ces progres, il faut que soient et demeurent unis l'effort individuel el l'effort collectif. Loin de moi la pensée de voufoir diminuer la valeur de l'initialive privée dont les industriels de la région ont domé de si grands exemples couronnés de si beaux résultats. Ii suis mème très heureux que le crédit, que l'on dit ehez nous si timido, ail frrmis d'engager des sonmes énormes pour la réalisation d'entreprises qui - par leur nature - doirent altendre pendant de longues annóes lour plein développement.

" I'Ptat ne saturait non plus se désintéresser de ces résultals. Ce serait manquer à son devoir enters une grande el nouvelle industric ; ce scrail onblirir, on outre, que les capitanx cugagés dans cette cuvre ont ou pom bujot la mise en valeur de forces nalure!les, de forces nationales. Il est Wom: lésirable pour tous que, dans les résultats comme dans la produclion. l'ktath reste l'associć des industricls, suirant une formule de collaboration cofulable, et ménageanl les intérèts privés.

"A Jouhy, (a) matin, on faisat appel an Ministre des Travaux publirs qui ne se dérobera pas ; les industriels pourraient également solliciler le's concours de la Chambre de Commere et de l'Université, dont je - wis pour quelques jours encore le Ministre intérimaire, pour la création l'tul laboratoire hydraulique. Ces conconrs ne leur seront pas refusés.

" lat collaboration dont jo parle peut être kgalement féconde si les industricts veulent hien signaler an Minietre des Travanx publics les modifiralions de procédure, les simplifications qui leur paraissent compatibles are li bnno instruction des affaires.

"An surplus. cette associntion cntre l'Etat ef l'industric n'est-clle pass déjà réaliséo ? J'ai été, non pas strpris, mais fier de voir la place yur tiennent, dans le développoment de cette industrie, les ingénieurs à lia tife desquels j'ai l'honneur d'ctre placé. On peut dire aves orgneil que, par les Rivoire-Vicat, les de In Brosse, les Wilhelm et d'autres, les intérêts généranx et les inlérêts privés sont bien servis.

"Le meilleur sourenir que j'emporte de cette tomrnée est d'avoir pu juger combien d'énergie, d'intelligence et d'activité se dépensent dans relle région au service des intérêts génćraux et pour le bien public. Jo n'exagère pas en dis ant que vous tous, industricls, en défendant les positions conquises, vous avez le sentiment de travailler non sculcment pour les maisons dont vous portez le drapeau, mais pour la France of le pays tont entier.

"Lo devoir du Gonvernement est de favoriser votre activité, et de vous permeltre d'obtenir le maximum d'intensité dans la production.

"Dans le ealme des vacances, des voix isolées se sont fait entendre nom se plaindre que l'on ne se batte pas assez. Plus que jamais le Gouvernement est dócidé à demeurer fidèle à sa devise, à sa méthode, au programme qui a reçu l'approbation du Parlement et dn Pays. Nous ne pensons pas que, s'il est désirable de maintenir la paix enfre les nations, il le soil moins de la maintenir entre les Français.

"Sans rien aliéner du programme nolitique et social, qui est le nôtre, f'slime, d'accord avec éminent. ami, M. le Président du Conseil, qu'il faut chereher à donnr au pays la paix et l'union nécossaires pour lui permellre, dans le travail ct dans la liberté, d'accroître sans cesse sa puissonce conomique et sa grandeur dans le monde.

\section{HYDRAULIQUE}

\section{AU PAYs DEs CHUTES DEAU}

La progression si rapide, en ces dernières années, du nombre et de l'importance des installations hydro-électriques dans les différents pays du monde, fait songer naturellement ì se poser cette question : "Quelles réserves d'énergie possćdons-nous encore dans les chutes d'eau restant à aménager ?"

Cette évaluation de la "houille blanche ", déjà utilisée ou demeurant encore disponible, est assez difficile à chiffrer, comme loute estimation échappant aux seules considérations théoriques. Cependant, de récentes statistiques résultant de travaux publiés par l'Électrical World et l'Electrolechnische Zeilschrifi, fourniraient les indications suivantes :

\begin{tabular}{|c|c|c|}
\hline & $\begin{array}{l}\text { Puissance totale } \\
\text { disponible }\end{array}$ & $\begin{array}{l}\text { Puissance utilisté } \\
\text { en } 1909\end{array}$ \\
\hline Anglelerre....... & $965.000 \mathrm{HP}$ & " \\
\hline Allemagne........ & 1.425 .900 & $294.400 \mathrm{HP}$ \\
\hline Suisse.......... & $x .500 .000$ & 380.000 \\
\hline Tlalic . . . . . . . . & 5.500 .000 & 464.000 \\
\hline France.......... & 5.857 .000 & 650.000 \\
\hline Autriche. . . . . . & 6.160 .000 & n \\
\hline Suède... . . . . & 6.750 .000 & $n$ \\
\hline Norvège. . . . . . . . & 7.500 .000 & $\eta$ \\
\hline Etats-Unis....... & $\eta$ & 5.250 .000 \\
\hline
\end{tabular}

De la comparaison de ces chiffres, il faudrait conclure que la proportion des forces utilisées sur leur tolalité existante serail de :
r $\%$ en France
$8,5 \%$ en Italie
$25 \%$ en Suisse
$20 \%$ cu $\Lambda$ llemagne

Mais la réalité ne nous parail pas devoir correspondre à celte estimation, qui laisserait espérer encore d'énormes réserves d'énergic, el l'évaluation ci-dessus de la puissance totale disponible dans les chutes d'eau des différents pays nous paraît plus théorique que pralique. Or, c'est le póint de vue pratique de cette question de la houille blanche qu'il faut envisager, sans se contenter évidemment d'un simple produit du débit (variable d'ailleurs) par une somme des différences de niveau successives.

l'utilisation de certaines forces hydrauliques peut, en effet, être rendue pratiquement impossible par des considéralions techniques ou économiques.

C'est ainsi que la configuration des montagnes ou teur structure, la valeur des terrains et les exigences de cerlains propriétaires, enfin d'autres obstacles encore, peuvent s'opposer à l'établissement de canaux de dérivation souterrains ou à découvert ; et, pour les grands cours d'eau de plaines, la plupart d'entre eux sont soumis aux nécessités de la navigation à laquelle des barrages pourraient porter préjudice, même avec le secours des écluses, impraticables d'ailleurs dans le cas de longues dérivations.

Le point de vue économique, d'autre part, n'est pas moins négligeable, car, souvent, l'immobilisation de capitaux fort considérables serait absolument déraisonnable en face de dépenses trop élevécs d'installation ou de transport de force à distance, qui ne laisseraient prévoir que des bénéfices nuls ou incertains.

Il faut donc se garder prudemment d'unc illusion trop facile sur la puissance disponible de l'énergie hydraulique' en réserve.

Prenons l'exemple de l'Italie, où cet écarl entre les prévisions et la réalité ćtait mis au point l'annće dernière dans un travail publié par le Giornale dei Lavori. On y montrait que les chevaux hydrauliques disponibles dans ce pays devaient être réduits à un peu plus de 2 millions, dont 7000 ooo ćtaient déjà concédés, et dont le tiers restant, après déduction de ces 700 ooo IIP, n'est pas utilisable, pour des raisons soit économiques, soit techniques, d'où il fallait conclure qu'il n'y a pas beaucoup plus de i million de chevaux restant encore disponibles et pratiquement utilisables en Ttalie.

Sans donner ici pour la France des comparaisons numériques, susceptibles de faire le sujel d'une étude spéciale, on peut déclarer, en toute certitude, que la réserve de forces 